\title{
On strong stability and stabilizability of systems of neutral type
}

\author{
R. Rabah ${ }^{1}$, G. M. Sklyar ${ }^{2}$, and A. V. Rezounenko ${ }^{3}$ \\ 1 IRCCyN UMR 6597, 1 rue de la Noë, BP 92101, F-44321 Nantes Cedex 3, \\ France. rabah@emn.fr \\ 2 Institute of Mathematics, University of Szczecin, 70-451 Szczecin, Wielkopolska \\ 15, Poland. sklar@sus.univ.szczecin.pl \\ 3 Department of Mechanics and Mathematics, Kharkov University, \\ 4 Svobody sqr., Kharkov, 61077, Ukraine. rezounenko@univer.kharkov.ua
}

\section{Introduction and preliminary results}

In this note we present a new approach for studying the strong stability and stabilizability properties of the functional differential equation of neutral type

$$
\dot{x}(t)=A_{-1} \dot{x}(t-1)+\int_{-1}^{0} A_{2}(\theta) \dot{x}(t+\theta) d \theta+\int_{-1}^{0} A_{3}(\theta) x(t+\theta) d \theta
$$

where $A_{-1}$ is constant $n \times n$-matrix, $\operatorname{det} A_{-1} \neq 0, A_{2}, A_{3}$ are $n \times n$-matrices whose elements belong to $L_{2}(-1,0)$.

This equation occurs, for example, when a system of neutral type is stabilized. Even if the initial system contains pointwise delays only, then the set of natural feedback laws contains distributed delays (see e.g., [15, 17]), so the corresponding closed-loop system takes the form (1).

The problem of exponential stability of systems like (1) is well studied $[8,10]$. Our purpose is to analyze more subtle properties of stability (and stabilizability), namely strong non-exponential asymptotic stability (see e.g. [4]). One needs to consider an operator model generated by the system (1) in some infinite dimensional space. It is well-known that for neutral type systems the choice of the phase-space is crucial (in contrast to the case of retarded functional differential equations where solutions are more smooth than the initial data).

In $[6,8]$, the framework is based on the description of neutral type systems in the space of continuous functions $C\left([-1,0] ; \mathbf{C}^{n}\right)$. The essential result in this framework is that the exponential stability is characterized by the condition that the spectrum of the system belongs to the open left-half plane. The problem of asymptotic (non-exponential) stability is much more complicated.

Following [25] we treat our system as a system in the Hilbert space $M_{2}=$ $\mathbf{C}^{n} \times L_{2}\left(-1,0 ; \mathbf{C}^{n}\right)$. This fact is important for us since it allows to use deep 
ideas and technique of the operator theory in Hilbert space $[1,7]$ and results [19] on the existence of Riesz basises (see Section 3 for more details) in the analysis the following operator model (see [25]) of the system (1)

$$
\frac{\mathrm{d}}{\mathrm{d} t}\left(\begin{array}{c}
y(t) \\
z_{t}(\cdot)
\end{array}\right)=\mathcal{A}\left(\begin{array}{c}
y(t) \\
z_{t}(\cdot)
\end{array}\right)=\left(\begin{array}{c}
\int_{-1}^{0} A_{2}(\theta) \dot{z}_{t}(\theta) \mathrm{d} \theta+\int_{-1}^{0} A_{3}(\theta) z_{t}(\theta) d \theta \\
\mathrm{d} z_{t}(\theta) / \mathrm{d} \theta
\end{array}\right),
$$

where the domain of $\mathcal{A}$ is given by

$$
\mathcal{D}(\mathcal{A})=\left\{(y, z(\cdot)): z \in H^{1}\left(-1,0 ; C^{n}\right), y=z(0)-A_{-1} z(-1)\right\} \subset M_{2} .
$$

Theorem 1. The operator $\mathcal{A}$ defined in (2) is the infinitesimal generator of a $C_{0}$-semigroup denoted $T(t) \equiv e^{\mathcal{A t}}, t \geq 0$ on the Hilbert space $M_{2}=\mathbf{C}^{n} \times$ $L_{2}\left(-1,0 ; \mathbf{C}^{n}\right)$.

If additionally $\operatorname{det} A_{-1} \neq 0$, then the operator $\mathcal{A}$ is the generator of a group $e^{\mathcal{A} t}, t \in \mathbf{R}$ on $M_{2}$.

Let us denote by $\mu_{1}, \ldots, \mu_{\ell}, \mu_{i} \neq \mu_{j}$ if $i \neq j$, the eigenvalues of $A_{-1}$ and the dimensions of their rootspaces by $p_{1}, \ldots, p_{\ell}, \sum_{k=1}^{\ell} p_{k}=n$. Consider the points $\lambda_{m}^{(k)} \equiv \ln \left|\mu_{m}\right|+i\left(\arg \mu_{m}+2 \pi k\right), m=1, . ., \ell ; k \in Z$ and the circles $L_{m}^{(k)}$ of fixed radius $r \leq r_{0} \equiv \frac{1}{3} \min \left\{\left|\lambda_{m}^{(k)}-\lambda_{i}^{(j)}\right|,(m, k) \neq(i, j)\right\}$ centered at $\lambda_{m}^{(k)}$.

Theorem 2. The spectrum of $\mathcal{A}$ consists of the eigenvalues only which are the roots of the equation $\operatorname{det} \Delta(\lambda)=0$, where

$$
\Delta_{\mathcal{A}}(\lambda)=\Delta(\lambda) \equiv-\lambda I+\lambda e^{-\lambda} A_{-1}+\lambda \int_{-1}^{0} e^{\lambda s} A_{2}(s) d s+\int_{-1}^{0} e^{\lambda s} A_{3}(s) d s
$$

The corresponding eigenvectors of $\mathcal{A}$ are $\varphi=\left(\begin{array}{c}C-e^{-\lambda} A_{-1} C \\ e^{\lambda \theta} C\end{array}\right)$, with $C \in$ $\operatorname{Ker} \Delta(\lambda)$.

There exists $N_{1}$ such that for any $k$, such that $|k| \geq N_{1}$, the total multiplicity of the roots of the equation $\operatorname{det} \Delta(\lambda)=0$, contained in the circle $L_{m}^{(k)}$, equals $p_{m}$.

To describe the location of the spectrum of $\mathcal{A}$ we use Rouche theorem. More precisely, for sufficiently large $k$ and any $m$ we show that $\left|f_{1}(\lambda)\right|>\left|f_{2}(\lambda)\right|$ for any $\lambda \in L_{m}^{(k)}$ and $f_{1}(\lambda) \equiv \operatorname{det}\left(A_{-1}-e^{-\lambda} I\right), f_{2}(\lambda) \equiv \operatorname{det}\left(A_{-1}-e^{-\lambda} I\right)-$ $\operatorname{det}\left(\left(A_{-1}-e^{-\lambda} I\right)+e^{\lambda} \int_{-1}^{0} e^{\lambda s} A_{2}(s) d s+e^{\lambda} \lambda^{-1} \int_{-1}^{0} e^{\lambda s} A_{3}(s) d s\right)$.

Thus, $f_{1}-f_{2}$ has the same number of roots inside $L_{m}^{(k)}$ as function $f_{1}$.

We start our analysis of the stability properties of the system (2), using the classical technique (see e.g. [5]), and prove the following

Theorem 3. Assume that the spectrum satisfies $\sigma(\mathcal{A}) \subset\{\lambda: \Re \lambda<0\}$ and

$$
\left\|A_{-1}\right\|+\left\|A_{2}\right\|_{L_{2}(-1,0)}<1 .
$$

Then the system (2) is exponentially stable. 
The proof of Theorem 3 is based on the fact that for a $C_{0}$-semigroup $T(t)$ on Hilbert space one has $s_{0}(A)=\omega_{0}(T)$, where $A$ is the generator of $T, s_{0}(A)$ is the abscissa of uniform boundedness of the resolvent and

$$
\omega_{0}(T) \equiv \inf \left\{\omega \in R: \exists M>0:\|T(t)\| \leq M e^{\omega t}, \forall t \geq 0\right\}
$$

See [24] for definitions and details. This fact is crucial for the following theorem which we use to prove Theorem 3.

Theorem 4. $[5$, p.222, th.5.1.5] Let $A$ be the infinitesimal generator of the $C_{0}$-semigroup $T(t)$ on Hilbert space $Z$. Then $T(t)$ is exponentially stable if and only if $(s I-A)^{-1} \in H_{\infty}(\mathcal{L}(Z))$. Here $H_{\infty}(\mathcal{L}(Z))$ is Hardy space of bounded holomorphic functions on $\{z \in C: \Re z>0\}$ with values in $\mathcal{L}(Z)$.

It is easy to see that Theorem 3 can not be applied to the case when there exists $\mu \in \sigma\left(A_{-1}\right)$ such that $|\mu|=1$ (see (4)). Moreover, Theorem 2 shows that under the assumptions of Theorem 3 one has $\sigma\left(A_{-1}\right) \subset\{\mu:|\mu| \leq \delta<1\}$ or equivalently $\sigma(\mathcal{A}) \subset\{\lambda: \Re \lambda \leq-\varepsilon<0\}$. For the system (1) this is the case of exponential stability.

Further on we are mainly interested in studying the case when the system is strongly asymptotically (non-exponentially) stable. Let us give an illustration of such a situation. We consider the scalar system (1)

$$
\dot{x}(t)=a \dot{x}(t-1)+\int_{-1}^{0} \varphi_{2}(\theta) \dot{x}(t+\theta) d \theta+\int_{-1}^{0} \varphi_{3}(\theta) x(t+\theta) d \theta
$$

where $a$ is a constant such that $|a|=1$, and $\varphi_{2}, \varphi_{3}$ are any functions belonging to $L_{2}(-1,0)$.

Proposition 1. The system (5) is strongly asymptotically (non-exponentially) stable if and only if $\sigma(\mathcal{A}) \subset\{\lambda: \Re \lambda<0\}$ (see (2) for the definition of $\mathcal{A}$ with $\varphi_{2}, \varphi_{3}$ instead of $\left.A_{2}, A_{3}\right)$.

To prove this and the other statements of this paper on the strong stability and stabilizability properties of neutral type systems we develop a new approach which we describe in details in the following sections.

First, we involve the classical theorem from the theory of semigroups (see Section 2) which is proposed as the main tool for the analysis of the stability properties. Second, we discuss a new result [19] on the existence of Riesz basises and, particularly, use it (see Section 3) to prove Proposition 1. Section 4 is devoted to the study of the stabilizability properties, when the control action is $B u(t)$. For neutral type systems, exponential stabilizability by feedback requires, in general, the delayed derivative in the feedback [15]. Our method allows to use only state feedback but we obtain asymptotic (non-exponential) stability of the closed loop system. To this end we recall first some deep results on strong stabilizability of linear systems in Hilbert spaces. In the last section one presents conclusions and perspectives. 


\section{Strong stability}

Apparently, the first result which may be considered as a basis of the investigation on strong stability is the following one [23, p.102].

Theorem 5. Let be given a complete nonunitary contraction $T$ in the Hilbert space $H$ such that

$$
\operatorname{mes}\left(\sigma(T) \cap S_{0}(1)\right)=0,
$$

where $S_{0}(1)=\{\lambda \in C:|\lambda|=1\}$ and mes(.) is a Lebesgue measure in $S_{0}(1)$. Then for each $x \in H$ we have

$$
\lim _{n \rightarrow \infty} T^{n} x=0 \quad \text { and } \quad \lim _{n \rightarrow \infty} T^{* n} x=0 .
$$

Let us recall that a contraction $T$ is said to be completely nonunitary if there does not exist a subspace $H_{1} \subset H$, invariant by $T$, such that $\left.T\right|_{H_{1}}$ is an unitary operator.

In the Theorem 5, the notion of stability is not explicitly involved. However, with this important theorem one can obtain the following result on asymptotic stability of a semigroup in Hilbert space.

Definition 1. A $C_{0}$-semigroup $T(t), t \geq 0$ is called to be contractive semigroup if $\|T(t)\| \leq 1, t \geq 0$.

Definition 2. $A$ semigroup $T(t), t \geq 0$ is said to be unitary if $\forall x \in H$ and $t \geq 0$ we have

$$
\|T(t) x\|=\|x\|=\left\|T(t)^{*} x\right\| .
$$

The semigroup $T(t)$ is said to be completely nonunitary if $\forall x \in H, x \neq 0$, there exists $t \geq 0$ such that

$$
\|T(t) x\|<\|x\| \quad \text { or } \quad\left\|T(t)^{*} x\right\|<\|x\| .
$$

Let us recall that a semigroup is contractive if and only if the infinitesimal generator $A$ of the semigroup is maximal dissipative: $\Re\langle A x, x\rangle \leq 0$ for all $x \in \mathcal{D}(A)$, and unitary if and only if the infinitesimal generator $A$ is skewadjoint.

Theorem 6. [12, §A3 Strong Stability of Evolution Equations]. Let A be the infinitesimal generator of a contractive completely nonunitary semigroup $e^{A t}, t \geq 0$ in the Hilbert space $H$ and

$$
\operatorname{mes}(\sigma(A) \cap(i \mathbf{R}))=0,
$$

where $i \mathbf{R}$ is the imaginary axis and mes(.) a Lebesgue measure on this set. Then for all $x \in H$ we have $e^{A t} x \rightarrow 0, \quad t \rightarrow \infty$. 
The proof of Theorem 6 is based on Theorem 5 and the introduction of the cogenerator of the semigroup [23], i.e. the operator

$$
F=(A+I)(A-I)^{-1} .
$$

The condition (6) is essential for the stability of the semigroup. For practical use, an important particular case is the condition: the set $(\sigma(A) \cap(i \mathbf{R}))$ is at most countable.

It turns out that when this condition is satisfied, then the semigroup $\left\{e^{A t}\right\}, t \geq 0$ is completely nonunitary if and only if the operator $A$ has no pure imaginary eigenvalues. This gives a simple formulation of the Theorem 6 .

Moreover, with this assumption the result on strong asymptotic stability may be extended to the case of Banach space. Namely one has the following criteria of strong asymtotic stability.

Theorem 7. Let $e^{A t}, t \geq 0$ be a $C_{0}$-semigroup in the Banach space $X$ and $A$ be the infinitesimal generator of the semigroup. Assume that $(\sigma(A) \cap(i \mathbf{R}))$ is at most countable and the operator $A^{*}$ has no pure imaginary eigenvalues. Then $e^{A t}$ is strongly asymptotically stable (i.e. $e^{A t} x \rightarrow 0, t \rightarrow+\infty$ as $x \in X$ ) if and only if one of the following conditions is valid:

i) There exists a norm $\|\cdot\|_{1}$, equivalent to the initial one $\|\cdot\|$, such that the semigroup $e^{A t}$ is contractive according to this norm: $\left\|e^{A t} x\right\|_{1} \leq\|x\|_{1}$, $\forall x \in X, t \geq 0$

ii) The semigroup $e^{A t}$ is uniformly bounded: $\exists C>0$ such that $\left\|e^{A t}\right\| \leq C$, $t \geq 0$.

The Theorem 7 was obtained first in [20] for the case of bounded operator $A$, then generalized in $[14,2]$ for the general case. The development of this theory concerns a large class of differential equations in Banach space (see [24] and references therein).

\section{Riesz basis property}

We notice that the condition " $\sigma(A) \cap(i \mathbf{R})$ is at most countable" (see Theorem 7) can be easily verified for many concrete systems which arise from applications. For example, in the case when $A$ has compact resolvent, one has that $\sigma(A)$ itself is at most countable and consists of the point spectrum only. The location of eigenvalues of $A$ for some systems can be easily described while for others this question needs a careful investigation, using, for example, Rouche theorem and perturbation analysis (see e.g. Theorem 2). It is well known that the property $\sigma(A) \subset\{\lambda: \Re \lambda<0\}$ is necessary but not sufficient for the strong asymptotic stability of $e^{A t}$.

Taking this into account, we arrive at the necessity to have an efficient method to check the property i) (or equivalently ii)) of Theorem 7. Working in a Hilbert space, we get a powerful tool to study the property i), namely, 
the concept of Riesz basis. The simplest case is a Riesz basis of vectors. Let us remind the definition.

Definition 3. A basis $\left\{\psi_{j}\right\}$ of a Hilbert space $H$ is called a Riesz basis if there are an orthonormal basis $\left\{\phi_{j}\right\}$ of $H$ and a linear bounded invertible operator $R$, such that $R \psi_{j}=\phi_{j}$.

To the best of our knowledge, the main source of abstract results on Riesz basises is the monograph [7]. The most desired situation for concrete systems is to have a Riesz basis formed by eigenvectors of $A$ or, at least, by generalized eigenvectors $[7,12,21]$. In more general situations, one studies the existence of basises formed by subspaces. We remind that a sequence of nonzero subspaces $\left\{V_{k}\right\}_{i}^{\infty}$ of the space $V$ is called basis (of subspaces) of the space $V$, if any vector $x \in V$ can be uniquely presented as $x=\sum_{k=1}^{\infty} x_{k}$, where $x_{k} \in V_{k}, k=1,2,$. . We say that the basis $\left\{V_{k}\right\}_{i}^{\infty}$ is orthogonal if $V_{i}$ is orthogonal to $V_{j}$ when $i \neq j$. As in the case of a basis of vectors we can introduce the following definition.

Definition 4. [7] A basis $\left\{V_{k}\right\}$ of subspaces is called a basis equivalent to orthogonal (a Riesz basis) if there are an orthogonal basis of subspaces $\left\{W_{k}\right\}$ and a linear bounded invertible operator $R$, such that $R V_{k}=W_{k}$.

The best "candidates" to form the basis of subspaces are generalized eigenspaces of the generator of a semigroup, but there are simple examples (see Example 1 below) showing that generalized eigenspaces do not form such a basis in the general case.

One of the crucial ideas of our approach is to construct a Riesz basis of finite-dimensional subspaces which are invariant for the generator of the semigroup (see (2)). The existence of such basises essentially simplifies, for example, the verification of the property i) of Theorem 7 .

In [19] we obtained the following general result.

Theorem 8. There exists a sequence of invariant for $\mathcal{A}$ (see (2)) finitedimensional subspaces which constitute a Riesz basis in $M_{2}$.

More precisely, these subspaces are $\left\{V_{m}^{(k)},|k| \geq N, m=1, . ., \ell\right\}$ and a $2(N+1) n$-dimensional subspace spanned by all eigen- and rootvectors, corresponding to all eigenvalues of $\mathcal{A}$, which are outside of all circles $L_{m}^{(k)}$, $|k| \geq N, m=1, . ., \ell$.

Here $V_{m}^{(k)} \equiv P_{m}^{(k)} M_{2}$, where

$$
P_{m}^{(k)} M_{2}=\frac{1}{2 \pi i} \int_{L_{m}^{(k)}} R(\mathcal{A}, \lambda) d \lambda
$$

are spectral projectors; $L_{m}^{(k)}$ are circles defined before.

We emphasize that the operator $\mathcal{A}$ may not possess in a Riesz basis of generalized eigenspaces. We illustrate this on the following 
Example 1. Consider the particular case of the system (1):

$$
\dot{x}(t)=A_{-1} \dot{x}(t-1)+A_{0} x(t), \quad A_{-1}=\left(\begin{array}{ll}
1 & 1 \\
0 & 1
\end{array}\right), \quad A_{0}=\left(\begin{array}{cc}
\alpha & 0 \\
0 & \beta
\end{array}\right) .
$$

One can check that the characteristic equation is $\operatorname{det} \Delta(\lambda)=\left(\alpha-\lambda+\lambda e^{-\lambda}\right)(\beta-$ $\left.\lambda+\lambda e^{-\lambda}\right)=0$ and for $\alpha \neq \beta$ there are two sequences of eigenvectors, such that $\left\|v_{n}^{1}-v_{n}^{2}\right\| \rightarrow 0$, as $n \rightarrow \infty$. By the definition, such vectors can not form a Riesz basis.

To prove Proposition 1 we notice that for the particular case of the system (5), Theorem 8 gives that $\ell=n=1$, all the subspaces $V_{1}^{(k)},|k| \geq N$, are one-dimensional and together with the $2(N+1)$-dimensional subspace form a Riesz basis of the space $M_{2}$. Using this property, we consider the operator $R$ (see Def. 3) which maps the eigen- and possibly finite number of root-vectors of $\mathcal{A}$ to an orthonormal basis of $M_{2}$. It is now easy to check that the new norm $\|\cdot\|_{1} \equiv\|R \cdot\|$ is equivalent to $\|\cdot\|$. This, together with the property $\sigma(\mathcal{A}) \subset\{\lambda: \Re \lambda<0\}$, allow us to apply Theorem 7 to prove Proposition 1 .

Let us precise that for the general multivariable system (1) the stability conditions is more complicated. A complete analysis for the general case will be given in one of our forcoming papers.

\section{Strong stabilizability}

\subsection{The abstract theory}

The problem of strong stabilizability of control systems in infinite dimensional spaces has been intensively studied since seventies. The basic abstract formulation of this problem is the following one.

Consider the linear system

$$
\dot{x}=A x+B u, \quad x \in H, u \in U,
$$

where $H, U$ are Hilbert spaces, the operator $A$ is the infinitesimal generator of a $C_{0}$-semigroup of contractions $\left\{e^{A t}\right\}, t \geq 0$, i.e. $\left\|e^{A t}\right\| \leq 1, t \geq 0$ or, what is the same, such that $A$ is a maximal dissipative operator. The operator $B$ is usually assumed to be a bounded linear operator from $U$ to $X$. The problem under investigation is: if the feedback control law $u=-B^{*} x$ is a stabilizing control, i.e. $e^{\left(A-B B^{*}\right) t} x \rightarrow 0, t \rightarrow+\infty, \forall x \in H$.

Under some additional assumptions this problem was studied in [22] using the Lyapunov method. In [13] and other works by N. Levan (cf. references in [16]) a framework based on the decomposition of the contractive semigroup and the harmonic analysis of operators was developed. Based on this framework the partial answers to the problem of strong stabilizability were given. 
Necessary and sufficient conditions of the strong stabilizability under the assumption (6) were given in the PhD thesis of G. M. Sklyar (Kharkov, 1983). In a slightly weaker formulation this result was published in [11]. The complete result was included to the book [12] (§A3. Strong Stabilizability of Evolution Equations). Let us recall it:

We denote for the system (8)

$$
L_{r}=\overline{\sum_{t \geq 0} e^{A t} B U} \quad \text { and } \quad L_{* r}=\overline{\sum_{t \geq 0} e^{A^{*} t} B U} .
$$

For the contractive semigroup $e^{A t}, t \geq 0$, the canonical decomposition [23] holds:

$$
H=V \oplus W,
$$

where the restriction $\left.e^{A t}\right|_{V}$ is an unitary semigroup, while the restriction $\left.e^{A t}\right|_{W}$ is completely nonunitary.

Theorem 9. Let for the system (8) the condition

$$
\operatorname{mes}(\sigma(A) \cap(i \mathbf{R}))=0
$$

be valid. Then the system (8) is strongly stabilizable if and only if

$$
V \cap L_{r}^{\perp} \cap L_{* r}^{\perp}=\{0\} .
$$

The stabilizing control law is then given by $u=-B^{*} x$.

Let us note that the condition (9) becomes much simpler when the set $(\sigma(A) \cap(i \mathbf{R}))$ is at most countable. It is equivalent to the following condition: There does not exist an eigenvector $x \in H$ of the operator $A$, with pure imaginary eigenvalue such that $B^{*} x=0$.

In this formulation the result was found one more time in [3], where the authors used Theorem 7. An extensive investigation of the strong stabilizability can be found [16] (see also references therein). In [21] the problem of the description of a large class of (strong) stabilizing control laws of the type $u=P x$ is given. The main tools are the Theorem 7 and the technique of the characterization of equivalent norms for which the operator $A+B P$ is dissipative. For the particular case when the operator $A$ is skew-adjoint with separated discrete spectrum this class was identified. The problem of robustness in this class was also investigated. The perspective is to develop this framework to the case of unbounded operator $P$ in the feedback $u=P x$.

The abstract theory of stabilizability given here may be applied to the system of neutral type. 


\subsection{Systems of neutral type}

Let us present some results on the stabilizability for the particular case of the system (2).

For simplicity we consider a control neutral type system with one delay in the state

$$
\dot{x}(t)=A_{0} x(t)+A_{1} x(t-1)+A_{-1} \dot{x}(t-1)+B u(t),
$$

$x \in R^{n}, u \in R^{r}, A_{j}, j=-1,0,1$ are $n \times n$-matrices, $B$ is a $n \times r$-matrix.

The stabilizability problem consists in determination of linear feedback control $u=p(x(\cdot))$ such that the closed-loop system

$$
\dot{x}(t)=A_{0} x(t)+A_{1} x(t-1)+A_{-1} \dot{x}(t-1)+B p(x(\cdot))
$$

becomes a stable one. The abstract functional model of the system (10) uses the operator $\mathcal{A}: \mathcal{D}(\mathcal{A}) \rightarrow M_{2}$ defined by (c.f. $\left.(2)\right)$

$$
\mathcal{A}\left(\begin{array}{c}
y \\
z(\cdot)
\end{array}\right)=\left(\begin{array}{c}
A_{0} y+\left(A_{1}+A_{0} A_{-1}\right) z(-1) \\
\frac{\partial}{\partial \theta} z(\cdot)
\end{array}\right)
$$

where $\mathcal{D}(\mathcal{A})$ is defined as before (see $(2))$.

With these notations (10) can be rewritten as

$$
\frac{d}{d t}\left(\begin{array}{c}
y(t) \\
z_{t}(\cdot)
\end{array}\right)=\mathcal{A}\left(\begin{array}{c}
y(t) \\
z_{t}(\cdot)
\end{array}\right)+\mathcal{B} u(t)
$$

where $\mathcal{B}=\left(\begin{array}{c}B \\ 0\end{array}\right)$ is a linear operator $\mathcal{B}: \mathbf{C}^{n} \rightarrow M_{2}$.

The spectrum $\sigma(\mathcal{A})$ is the set

$$
\sigma(\mathcal{A})=\sigma=\left\{\lambda \mid \operatorname{det}\left(\lambda I-A_{-1} \lambda e^{-\lambda}-A_{0}-A_{1} e^{-\lambda}\right)\right\}=0 .
$$

and consists of eigenvalues only. Denote further by $\sum$ the set of all nonzero eigenvalues of matrix $A_{-1}$. Then for any $\mu \in \sum$ the set $\sigma$ includes a family of eigenvalues

$$
\sum^{\mu}=\left\{\lambda_{k}^{\mu}=\log |\mu|+i(\operatorname{Arg} \mu+2 \pi k)+\bar{o}(1), \quad k \in \mathbf{Z}\right\}
$$

where $\bar{o}$ is meant as $k \rightarrow \pm \infty$.

We assume that the following assumptions are satisfied.

(a1) $\sum \subset\{w:|w| \leq 1\}$ and there exists $\mu \in \sum:|\mu|=1$.

(a2) All the eigenvalues $\mu \in \sum$ such that $|\mu|=1$ are simple in the sense that there are no Jordan chains corresponding to such eigenvalues.

(a3) Finite-dimensional system

$$
\dot{x}(t)=A_{0} x(t)+B u(t), \quad x \in R^{n}, u \in R^{r}
$$


is controlable, i.e. $\operatorname{rank}\left(B, A_{0} B, \ldots, A_{0}^{n-1} B\right)=n$. In particular, this means that (13) is stabilizable, i.e. there exists a linear feedback control $u=P_{0}^{0} x$ such that $\Re \sigma\left(A_{0}+B P_{0}^{0}\right)<0$.

(a4) $\operatorname{rank}\left(A_{1}+A_{0} A_{-1}, B\right)=\operatorname{rank} B$.

As it is discussed in [18], we are mainly interested in the controls which are bounded with respect to operator $\mathcal{A}$ (for the definition and details see e.g. [9]) i.e. controls of the form

$$
u=\mathcal{P}(x(\cdot))=\int_{-1}^{0} \tilde{P}(\theta) \dot{x}(t+\theta) d \theta+\int_{-1}^{0} \hat{P}(\theta) x(t+\theta) d \theta,
$$

where $\tilde{P}(\theta), \hat{P}(\theta), \theta \in[-1,0]$ are square-integrable $(r \times n)$-matrix-functions. This is the natural choice of controls to achieve the non-exponential stabilizability.

We have the following result.

Theorem 10. Let the system (10) satisfy the assumptions (a1) - (a4). Then this system is strongly stabilizable with the aid of feedback controls which are bounded with respect to operator $\mathcal{A}$ if and only if for an arbitrarily chosen matrix $P_{0}$ such that

$$
\sigma\left(A_{0}+B P_{0}\right) \cap \log \left(\sum\right) \cap(i \mathbf{R})=\emptyset
$$

there do not exist an eigenvector $g$ of $A_{-1}$ corresponding to an eigenvalue $\mu \in \sum,|\mu|=1$ and $k \in \mathbf{Z}$ such that

$$
B^{*} R_{\lambda_{k}^{\mu}}^{*}\left(A_{0}+B P_{0}\right) g=0,
$$

where $\lambda_{k}^{\mu}$ is given by (12). Under this condition the strong stabilization can be achieved by the choice of control:

$$
u=Q_{1} x(t)+Q_{2} x(t-1)+\int_{-1}^{0} Q_{3}(\theta) x(\theta) d \theta,
$$

where $Q_{1}, Q_{2}$ are constant $(r \times n)$-matrices, $Q_{3}$ is $(r \times n)$-matrix which elements belong to $L_{2}(-1,0)$.

The proof is based on the analysis of the system (11) and using the Theorem 9.

\section{Conclusions and perspectives}

In this note we presented a new approach for investigation the strong stability and stabilizability for systems of neutral type. We describe the main ideas and facts which form the background of our research. These facts are collected from 
the theory of differential equation with retarded argument (see e.g. [5, 6, 8]), the theory of semigroups as well as general operator theory (see e.g. [7, 9, 23, $24]$ and recent results from $[18,19])$. We give examples emphasizing that our approach is widely applicable, perspective and extends the classical stability theory.

Acknowledgements. This work was realized with the financial support of Region Pays de la Loire, Ecole Centrale de Nantes (France) and Polish KBN Grant 5 PO3A 03021.

\section{References}

1. Akhiezer N. I., Glazman I. M. (1993) Theory of linear operators in Hilbert space. Translated from the Russian and with a preface by Merlynd Nestell. Reprint of the 1961 and 1963 translations. Two volumes. Dover Publications, New York.

Theory of linear operators in Hilbert space. Vol. I, II. Transl. from the 3rd Russian ed. Monographs and Studies in Mathematics, 9, 10. Edinburgh. Boston - London -Melbourne: Pitman Advanced Publishing Program. XXXII, 552 p.

2. Arendt W., Batty C. J. (1988) Tauberian theorems and stability of oneparameter semigrouos. Trans. Amer. Math. Soc. 306:837-852

3. Batty C. J., Phong V. Q. (1990) Stability of individual elements under oneparameter semigroups. Trans. Amer. Math. Soc. 322:805-818.

4. Brumley W. E. (1970) On the asymptotic behavior of solutions of differentialdifference equations of neutral type. J. Differential Equations 7, 175-188.

5. Curtain R. F., Zwart H. (1995) An introduction to infinite-dimensional linear systems theory. Springer-Verlag, New York.

6. Diekmann O, van Gils S, Verduyn Lunel S. M., Walther H-O (1995) Delay equations. Functional, complex, and nonlinear analysis. Applied Mathematical Sciences, 110. Springer-Verlag, New York.

7. Gohberg I. C., Krein M. G. (1969) Introduction to the theory of linear nonselfadjoint operators (English) Translations of Mathematical Monographs. 18. Providence, RI: AMS. XV, 378 p.

8. Hale J., Verduyn Lunel S. M. (1993) Theory of functional differential equations. Springer-Verlag, New York.

9. Kato T (1980) Perturbation theory for linear operators. Springer Verlag.

10. Kolmanovskii V, Myshkis A (1999) Introduction to the theory and applications of functional differential equations., Mathematics and its Applications (Dordrecht). 463. Dordrecht: Kluwer Academic Publishers.

11. Korobov V. I., Sklyar G. M. (1984) Strong stabilizability of contractive systems in Hilbert space. Differentsial'nye Uravn. 20:1862-1869.

12. Krabs W., Sklyar G. M. (2002) On Controllability of Linear Vibrations. Nova Science Publ. Huntigton, N.Y.

13. Levan N., Rigby I. (1979) Strong stabilizability of linear contractive systems in Banach space. SIAM J. Control, 17:23-35.

14. Lyubich Yu. I., Phong V. Q. (1988) Asymptotic stability of linear differential equation in Banach space. Studia Math. 88:37-42. 
15. O'Connor D. A, Tarn T. J. (1983) On stabilization by state feedback for neutral differential equations. IEEE Transactions on Automatic Control. Vol. AC-28, n. $5,615-618$.

16. Oostvenn J (1999) Strongly stabilizable infinite dimensional systems. Ph.D. Thesis. University of Groningen.

17. Pandolfi L. (1976) Stabilization of neutral functional differential equations. J. Optimization Theory and Appl. 20, n. 2, 191-204.

18. Rabah R., Sklyar G. M., On a class of strongly stabilizable systems of neutral type, (submitted).

19. Rabah R., Sklyar G. M., and Rezounenko A.V (2003) Generalized Riesz basis property in the analysis of neutral type systems, Comptes Rendus de l'Académie des Sciences, Série Mathématiques. To appear. (See also the extended version, Preprint RI02-10, IRCCyN, Nantes, France).

20. Sklyar G.M., Shirman V.Ya. (1982) On Asymptotic Stability of Linear Differential Equation in Banach Space. Teor, Funk., Funkt. Analiz. Prilozh. 37: 127-132.

21. Sklyar G, Rezounenko A. (2001) A theorem on the strong asymptotic stability and determination of stabilizing control. C.R.Acad. Sci. Paris, Ser. I. 333:807812 .

22. Slemrod M (1973) A note on complete controllability and stabilizability for linear control systems in Hilbert space. SIAM J. Control 12:500-508.

23. Sz.-Nagy, B., Foias, C. (1970) Harmonic Analysis of Operators on Hilbert Space. Budapest: Akadémiai Kiadó; Amsterdam-London: North-Holland Publishing Company. XIII, 387 p.

24. van Neerven J. (1996) The asymptotic behaviour of semigroups of linear operators, in "Operator Theory: Advances and Applications", Vol. 88. Basel: Birkhauser.

25. Yamamoto Y, Ueshima S (1986) A new model for neutral delay-differential systems. Internat. J. Control 43(2):465-471. 